\title{
Masses of X-ray Pulsars in Binary Systems with OB Supergiants
}

\author{
M. K. Abubekerov ${ }^{1}$, É. A. Antokhina ${ }^{2}$, and A. M. Cherepashchuk ${ }^{1,2}$ \\ ${ }^{1}$ Moscow State University, Vorob'evy gory, Moscow, 119992 Russia \\ ${ }^{2}$ Sternberg Astronomical Institute, Universitetskiü pr. 13, Moscow, 119992 Russia \\ Received June 15, 2003; in final form, August 8, 2003
}

\begin{abstract}
We describe the results of a statistical approach to analyzing the combined radial-velocity curves of X-ray binaries with OB supergiants in a Roche model, both with and without allowance for the anisotropy of the stellar wind. We present new mass estimates for the $\mathrm{X}$-ray pulsars in the close binary systems Cen X-3, LMC X-4, SMC X-1, 4U 1538-52, and Vela X-1. (C) 2004 MAIK “Nauka/Interperiodica".
\end{abstract}

\section{INTRODUCTION}

Neutron stars are a natural product of the evolution of stars with masses in excess of $10 M_{\odot}$. They were predicted theoretically by Landau [1] in the early 1930s, and the first radio pulsar was found in the center of the Crab nebula in the early 1960s. At the beginning of the 1970s - an epoch of active programs in near-Earth space exploration-the "Uhuru" satellite discovered objects with periodic X-ray variability [2] (X-ray pulsars), later identified with neutron stars in binary systems.

Thousands of compact X-ray sources have now been discovered in our Galaxy and other nearby galaxies. Most of these are close binary systems, in which the optical component supplies matter to the neutron star. Accretion with subrelativistic velocities onto the compact component's surface releases immense amounts of $\mathrm{X}$-ray energy with luminosities of the order of $10^{36}-10^{39} \mathrm{erg} / \mathrm{s}$ [3-7]. From the point of view of the equation of state of the superdense matter, the key characteristic of an X-ray pulsar is its mass. The theory predicts two stable modes for neutron stars, based on a "soft" equation of state, with $M_{\max } \simeq 1.4 M_{\odot}$, and a "hard" equation of state, with $M_{\max } \simeq 1.8 M_{\odot}$ [8-12]. The question of whether or not we should adopt the hard equation of state for neutron stars remains unanswered. Among known $\mathrm{X}$-ray pulsars, only Vela $\mathrm{X}-1$ has a mass close to $1.8 M_{\odot}$ [13]. Studies of X-ray binaries have been carried out for more than 30 years. Extensive spectroscopic observations have been accumulated which not only enable but also demand for their interpretation a more accurate allowance for effects resulting from the closeness of the components. A point-mass model approximating the optical component as a source of electromagnetic radiation with infinitesimal size is not adequate for analysis of the rich observational data that are now available and cannot be used to correctly estimate the masses of the $\mathrm{X}$-ray pulsars in binary systems. This is especially true for systems with OB supergiants.

In X-ray binaries with OB supergiants, the optical star nearly fills its critical Roche lobe, which determines the limiting equilibrium figure in a rotating reference frame fixed to the binary. Therefore, the shape of the optical component deviates from a sphere, and the velocity field at the stellar surface becomes strongly nonuniform. The surface of the star that is turned toward the X-ray pulsar is heated by the pulsar's radiation, and the nonuniform gravitational acceleration on the companion leads to anisotropy of the stellar wind. All these factors distort the radialvelocity curve of the optical star. The distortions of the radial-velocity curve due to the effects of outflow and the stellar wind in close binaries with $\mathrm{OB}$ supergiants are comparable to the orbital velocity. These facts show the necessity of proper fitting of close-binary radial-velocity curves in a Roche model taking into account the anisotropy of the stellar wind [14].

\section{DESCRIPTION OF THE ROCHE MODEL}

The radial-velocity curves of $\mathrm{X}$-ray binaries are usually analyzed using models with two point masses, though it is not appropriate to approximate the stars in most close binaries as point masses moving in Keplerian orbits for the following reasons.

1. Due to tidal deformation, the optical component is ellipsoidal or pear-shaped due to the gravitational action of the relativistic companion, leading to strongly nonuniform surface temperatures (gravitational darkening). For mass ratios $q=m_{x} / m_{v}<1$ (with $m_{x}$ and $m_{v}$ being the masses of the optical and X-ray components), the binary centers of mass 
are inside the body of the optical star, leading to significant distortion of the radial-velocity curve.

2. X-ray heating of the side of the optical star facing the relativistic component results in a nonuniform temperature distribution over the stellar surface.

3. It is important for early-type stars in close binaries that the cores of strong absorption lines formed in the outermost atmospheric layers, at the base of the stellar wind, experience radial Doppler shifts. These shifts are due to the regular outflow of the stellar-wind plasma with velocities of the order of the sound speed, $V_{s} \sim T^{1 / 2}$. The temperature and local gravitational acceleration vary strongly at the surface of the tidally deformed star, leading to appreciable distortion of the optical component's radial-velocity curve. For earlytype stars, the velocity of the stellar wind at its base becomes comparable to the orbital velocity of the optical component, of the order of $10-20 \mathrm{~km} / \mathrm{s}$.

For these reasons, the radial-velocity curves of $\mathrm{X}$-ray binaries with $\mathrm{OB}$ supergiants should be analyzed using Roche models. We applied our algorithm for synthesizing light curves, radial-velocity curves, and absorption-line profiles, described in detail in $[15,16]$, to analyze observations of massive close X-ray binaries. We describe the optical star using a Roche model, treating its companion as a point X-ray source. We briefly summarize the main principles of our algorithm below.

The optical star and point relativistic object with masses of $m_{v}$ and $m_{x}$ move in elliptical orbits about their common center of mass. The inclination of the binary orbit to the plane of the sky is $i$. In general, the star's axial rotation is not assumed to be synchronous with the orbital motion. The star's shape coincides with an equipotential surface of the Roche model [16, 17]. The size of the star is determined by the filling coefficient for the critical Roche lobe, $\mu=R / R^{*}$, where $R$ and $R^{*}$ are the polar radii for partial and complete filling of the critical Roche lobe at the orbit periastron. The star's tidally distorted surface is subdivided into thousands of mass elements, with the emergent radiation computed for each of them, after which the contributions of the various areas are added taking into account the visibility of each area element at various phases of the orbital period. The computed radiation fluxes from each area element include the effects of gravitational darkening, heating of the stellar surface by the radiation from the companion (the "reflection" effect), and limb darkening. The absorption line profile and line equivalent width for each visible area with its temperature $T$ and local gravitational acceleration $g$ were calculated using the tables of Kurucz [18] for the Balmer lines and an interpolation procedure. We add the local profiles over the stellar surface after first normalizing to the continuum for each area, taking into account the Doppler effect to obtain the total profile for the star at the corresponding phase of the orbital period. The theoretical total profile is subsequently used to determine the star's radial velocity and derive the radial-velocity curve over the course of the orbital period (for more details, see [15]).

The input parameters of the algorithm used to compute the radial-velocity curve of an X-ray binary in the Roche model are collected in Table 1.

\section{COMPARISON OF THE THEORETICAL RADIAL-VELOCITY CURVES IN THE ROCHE AND POINT-MASS MODELS}

Our main goal was to refine available estimates of the masses of X-ray pulsars in close binaries. Before analyzing the observations, we carried out test computations to compare the theoretical radial-velocity curves in the Roche and point-mass models. We computed the radial-velocity curves using the $\mathrm{H} \gamma$ line for a hypothetical $\mathrm{X}$-ray system with parameters ( Table 1$)$ close to those of the studied systems: Cen X-3, LMC X-4, SMC X-1, Vela X-1, and 4U 1538-52 (Table 2).

To identify the influence of reflection effects on the radial-velocity curves, we computed them in the Roche model for low and high incoming $\mathrm{X}$-ray fluxes, $k_{x}=0.05$ and $k_{x}=1.4$. The computations of the radial-velocity curves from the $\mathrm{H} \gamma$ lines demonstrate that the semiamplitude of the curves decreases in the presence of stronger X-ray heating; a similar result was obtained earlier [15]. We can explain this by the fact that, in the case of a stronger $\mathrm{X}$-ray flux incident on the star and stronger heating of the surface facing the companion, the contribution of the light from the star's "nose" to the combined luminosity increases. The areas on the nose contribute more strongly to the star's mean radial velocity. The velocities of such areas are lower than the star's center-of-mass velocity, and so the mean velocity of the star decreases.

The difference of the star's radial-velocity curve from the curve for its center of mass increases from phase 0.0 to phase 0.5 as the star moves along its orbit and its heated surface turns toward the observer. At phase 0.0 , the star is in front of the $\mathrm{X}$-ray source. The difference between the star's radial-velocity curves in the Roche and point-mass models should increase with decreasing $q=m_{x} / m_{v}$.

Figure 1a compares the theoretical radial-velocity curves of a system (for its parameters, see Table 1) in the Roche and point-mass models. Curves are plotted for the $\mathrm{H} \gamma$ line and for the case of low heating, $k_{x}=0.05$. The semiamplitude of the curve is lower in the Roche model, and the semiamplitudes of the radial-velocity curves can be the same only when the 
Table 1. Numerical parameters used to synthesize an X-ray binary radial-velocity curve in the Roche model

\begin{tabular}{l|c|c}
\hline Relativistic component's mass & $m_{x}, M_{\odot}$ & 1.35 \\
Optical component's mass & $m_{v}, M_{\odot}$ & 20 \\
Period & $P$, days & 4 \\
Eccentricity & $e$ & 0 \\
Longitude of periastron of the optical component & $\omega$, degrees & 0 \\
Radial velocity of the system's center of mass & $V_{\gamma}, \mathrm{km} / \mathrm{s}$ & 0 \\
Orbital inclination & $i$, degrees & 80 \\
Roche-lobe filling coefficient for the optical component & & \\
at periastron & $f$ & 0.99 \\
Rotational asynchronism coefficient & $T_{e f f}, \mathrm{~K}$ & 0.95 \\
Effective temperature of the optical component & $\beta$ & 30000 \\
Gravitational darkening coefficient & & 0.25 \\
Ratio of the relativistic component's X-ray luminosity & $k_{x}=L_{x} / L_{v}$ \\
to the optical component's bolometric luminosity & $A$ & 0.05 и 1.4 \\
Coefficient for reprocessing of the X-ray radiation & $u$ & 0.5 \\
Limb-darkening coefficient & & 0.3 \\
\hline
\end{tabular}

Table 2. Observed characteristics of X-ray binaries with OB supergiants (from the literature [13, 19, 20])

\begin{tabular}{l|l|c|l|c|c|c|c|c}
\hline \multicolumn{1}{c|}{ Name } & Spectral type & $T_{\text {eff }}, \mathrm{K}$ & \multicolumn{1}{c|}{$P$, days } & $e$ & $i$, degrees & $\mu$ & $f$ & $K_{x}, \mathrm{~km} / \mathrm{s}$ \\
\hline Cen X-3 & O(6-9) II-III & 35000 & 2.0871390 & $<0.0008$ & $83_{-3}^{+3}$ & $0.995_{-0.005}^{+0.005}$ & $0.95_{-0.25}^{+0.27}$ & $414.1 \pm 0.9$ \\
LMC X-4 & O7 III-V & 35000 & 1.40839 & $<0.01$ & $63_{-3}^{+3}$ & $\sim 1.0$ & $0.65_{-0.19}^{+0.23}$ & 400.6 \\
SMC X-1 & B0.5Ia & 25000 & 3.89229118 & $<0.00004$ & $65_{-5}^{+5}$ & $0.97_{-0.03}^{+0.03}$ & $0.95_{-0.27}^{+0.34}$ & $301.5 \pm 4$ \\
Vela X-1 & B0.5Ibeq & 25000 & 8.964368 & 0.0898 & $73_{-3}^{+3}$ & $0.95_{-0.04}^{+0.04}$ & $0.69_{-0.08}^{+0.09}$ & $278.1 \pm 0.3$ \\
4U 1538-52 & B0Iabe & 25000 & 3.72844 & 0.08 & $60_{-5}^{+5}$ & $0.95_{-0.04}^{+0.04}$ & $0.94_{-0.25}^{+0.32}$ & $309.0 \pm 11$ \\
\hline
\end{tabular}

The orbital inclination $i$ was derived from the duration of the X-ray eclipse (see, e.g., [20]). The values of $e$ and $K_{x}$ were derived from the X-ray pulsar's radial-velocity curve.

mass of the relativistic component is increased from $1.35 M_{\odot}$ to $1.45 M_{\odot}$. To obtain equal semiamplitudes for phase 0.25 , the mass of the X-ray pulsar must be increased to $1.55 M_{\odot}$. For stronger heating, $k_{x}=$ 1.4, with the remaining parameters unchanged, equal semiamplitudes in the Roche and point-mass models require that the $\mathrm{X}$-ray pulsar's mass be increased to $1.65 M_{\odot}$.

Thus, all other conditions being the same, the use of the Roche model leads to an increase in the mass of the relativistic component by $\sim 7-20 \%$ compared to the mass obtained in the point-mass model. This suggests that using the more realistic close-binary model to analyze the radial-velocity curves should enable us to determine whether the masses of the neutron stars in X-ray binaries with $\mathrm{OB}$ supergiants exceed the masses of radio pulsars, which are equal, on average, to $1.35 \pm 0.04 M_{\odot}[21]$.

\section{OBSERVATIONAL MATERIAL}

Five eclipsing $\mathrm{X}$-ray binaries with OB supergiants have been discovered to date (Table 2). During the last 30 years, extensive observational material has been accumulated for these systems, making it possible to test hypotheses using statistical methods. It is not possible to correctly take into consideration the components' mutual influence with the widely used 


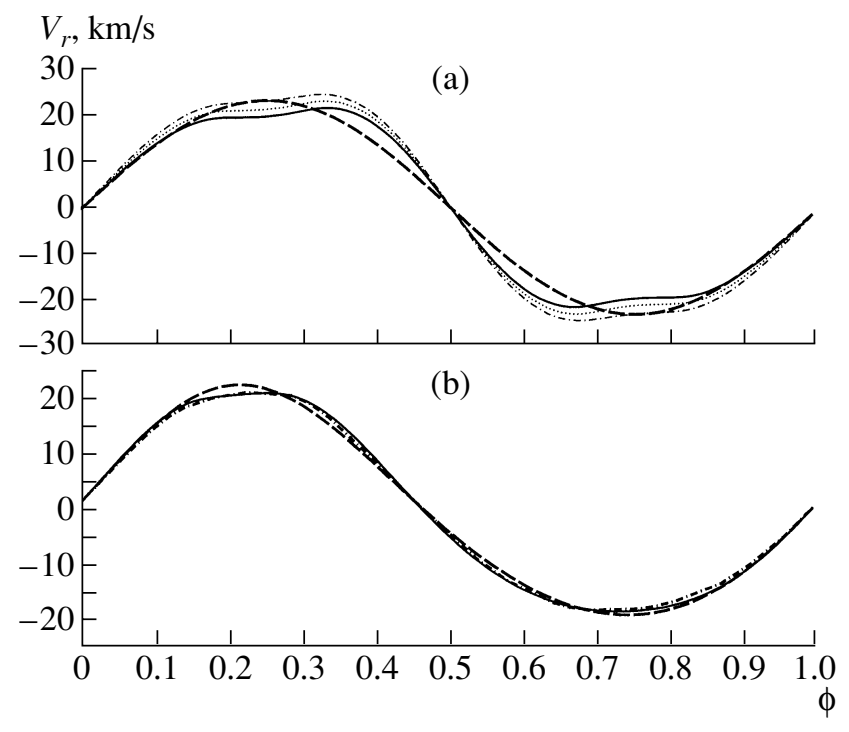

Fig. 1. (a) Theoretical radial-velocity curves of the optical star in a hypothetical X-ray close binary (for its parameters, see Table 1). The curves are derived from the $\mathrm{H} \gamma$ line. The dashed curve corresponds to the optical star in a point-mass model with $m_{x}=1.35 M_{\odot}$, and the solid curve, to a Roche model with $m_{x}=1.35 M_{\odot}$; the dotted curve corresponds to $m_{x}=1.45 M_{\odot}$, and the dot-dash curve, to $m_{x}=1.55 M_{\odot}$. (b) Comparison of the theoretical radial-velocity curves of a star in the Roche model based on the $\mathrm{H} \gamma$ line (solid curve) and the HeI $4471 \AA$ line (dot-dash curve). The parameters are close to those of Vela X-1 $\left(m_{v}=23 M_{\odot}, m_{x}=\right.$ $1.8 M_{\odot}, P_{\text {orb }}=8.96$ days, $e=0.0898, \omega=332.59^{\circ}, i=$ $73^{\circ}, \mu=0.99, f=0.69, T=25000 \mathrm{~K}, \beta=0.25, k_{x}=$ $0.05, A=0.5, u=0.38$ ). The dashed curve is the radialvelocity curve for the optical star's center of mass.

Monte Carlo method in the framework of the pointmass model. For the reasons indicated above, earlier estimates of the masses of X-ray pulsars must be refined and revised using the Roche model.

We constructed a master radial-velocity curve for each of the systems. We describe the spectroscopic data used for each radial-velocity curve below. The center of the X-ray eclipse was taken as the zero phase for all the systems.

Cen X-3. The system consists of an $\mathrm{O}$ giant (O(6-9)II-III), with mass $17-18 M_{\odot}$ and the X-ray pulsar, and has an orbital period of 2.0871390 days. The orbital eccentricity derived from timing of the $\mathrm{X}$-ray pulses is very low $(e<0.0008$ [13]), and we have assumed a circular orbit.

We used data from [22-24] acquired in 19761997 to construct the master radial-velocity curve (Fig. 2a). The data of [23] were corrected only for the close binary's $\gamma$ velocity. The corrections for the velocity of the stellar wind were already introduced by the authors when combining the mean

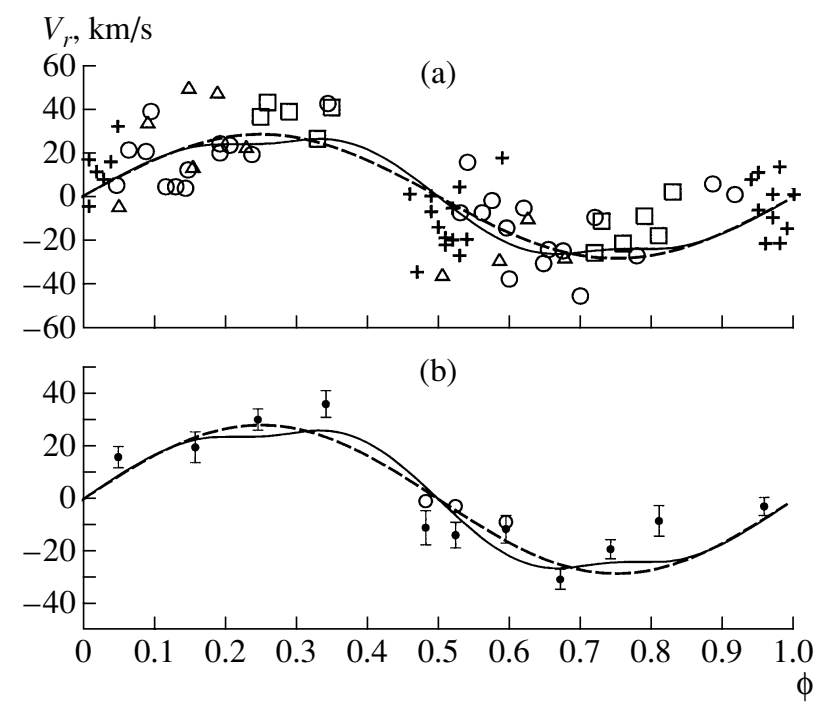

Fig. 2. (a) Master observed radial-velocity curve for the close X-ray binary Cen X-3 (triangles show spectroscopic data for 1976 from [22]; circles, data for 1978 from [23]; crosses, data for 1990 from [24]; and squares, data for 1997 from [24]). For comparison, the theoretical radial-velocity curves are shown for the Roche model (solid curve) and point-mass model (dashed curve), with $m_{x}=1.22 M_{\odot}$ corresponding to the minimum residual for the Roche model computed using method 2. The parameters are collected in Table 3. (b) Radial velocities averaged within phase intervals (filled circles are the mean radial velocities for the phase bins, and open circles, the radial velocities corrected for the normalized anisotropy function of the optical star's wind). The theoretical radialvelocity curves for the Roche model (solid curve) and point-mass model (dashed curve) with $m_{x}=1.22 M_{\odot}$ are shown for comparison.

observed radial velocities derived from the shifts of the HeI $4471 \AA$, HeI $5875 \AA$ absorption lines and the hydrogen Balmer lines. The line shifts in [22, 24] were derived relative to the spectrum averaged over all phases, so that the $\gamma$ velocity was taken into account in the method itself. The mean radial velocities presented in [22] were derived from the HeI $4471 \AA$, HeII $4541 \AA$ absorption lines and the hydrogen Balmer lines. Of the radial velocities from [24], we used only those measured for the $\mathrm{H} \gamma$ line. A total of 79 data points were available to us, distributed approximately uniformly in phase. We used these data to construct a master radial-velocity curve for the Cen X-3 system from the $\mathrm{H} \beta, \mathrm{H} \gamma, \mathrm{H} \delta$, HeI $4471 \AA$, and HeI $5875 \AA$ absorption lines. Our test computations demonstrated that the theoretical radial-velocity curves for the Roche model based on $\mathrm{H} \gamma$ and other lines $(\mathrm{H} \beta, \mathrm{H} \delta$, HeI $4471 \AA$ ) coincided, all other parameters being the same (Fig. 1b; see also the text below). Thus, we used the theoretical radialvelocity curves for the $\mathrm{H} \gamma$ line when fitting the master 


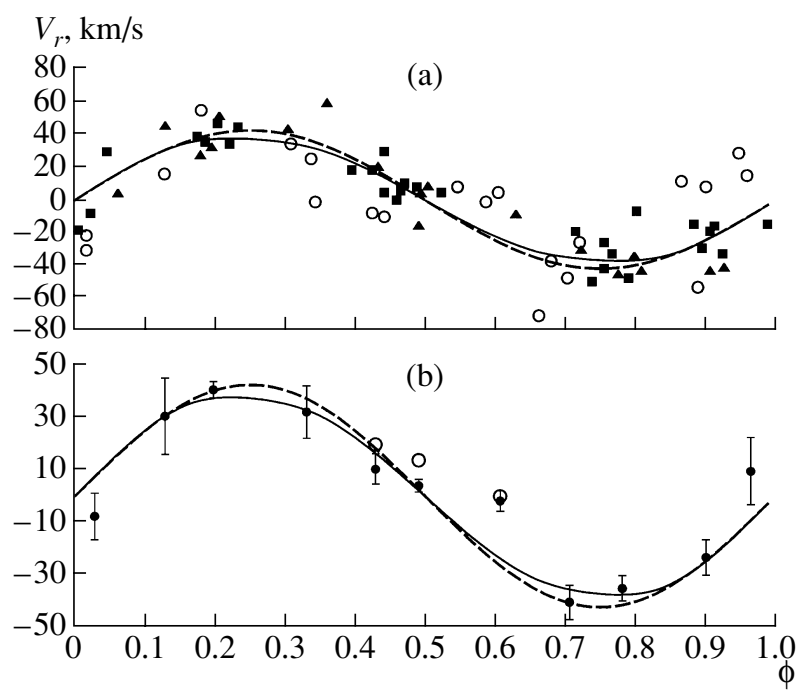

Fig. 3. (a) Master observed radial-velocity curve for the close X-ray binary LMC X-4 (filled triangles show spectroscopic data for 1975 from [25]; circles and filled squares show spectroscopic data for 1982 from [26] acquired with 2.5-m and 4-m telescopes). For comparison, theoretical radial-velocity curves are shown for the Roche model (solid curve) and point-mass model (dashed curve), with $m_{x}=1.63 M_{\odot}$ corresponding to the minimum residual for the Roche model computed using method 2. The parameters are collected in Table 3. (b) Radial velocities averaged within phase intervals (filled circles are the mean radial velocities for the phase bins, and open circles, the radial velocities corrected for the normalized anisotropy function of the optical star's wind). For comparison, theoretical radial-velocity curves are shown for the Roche model (solid curve) and point-mass model (dashed curve) with $m_{x}=1.63 M_{\odot}$.

radial-velocity curves for Cen $\mathrm{X}-3$, as well as for the other close binaries. The system's $\gamma$ velocity derived using the spectroscopic data of [23] is $39 \mathrm{~km} / \mathrm{s}$.

LMC X-4. The system consists of the X-ray pulsar and an optical star of spectral type O7III-V with a mass of $14-15 M_{\odot}$. The eccentricity is very low $(e<0.01)$, and we assumed the orbit to be circular. The orbital period derived from studies of the X-ray pulsar is 1.40839 days.

We used the data of $[25,26]$ acquired in 19751982 to plot the master light curve (Fig. 3a). The earlier observational data presented in [25] were already used in [26]. Thus, we used the newest spectroscopic data of [26]. The radial velocities were derived from the hydrogen absorption lines. Prior to being included in the master radial-velocity curves, the data were corrected for the $\gamma$ velocity in [26]. The system's $\gamma$ velocity derived using the spectroscopic data of [26] is $275 \mathrm{~km} / \mathrm{s}$.

SMC X-1. The system contains an optical component of spectral type B0.5Ia with a mass of about

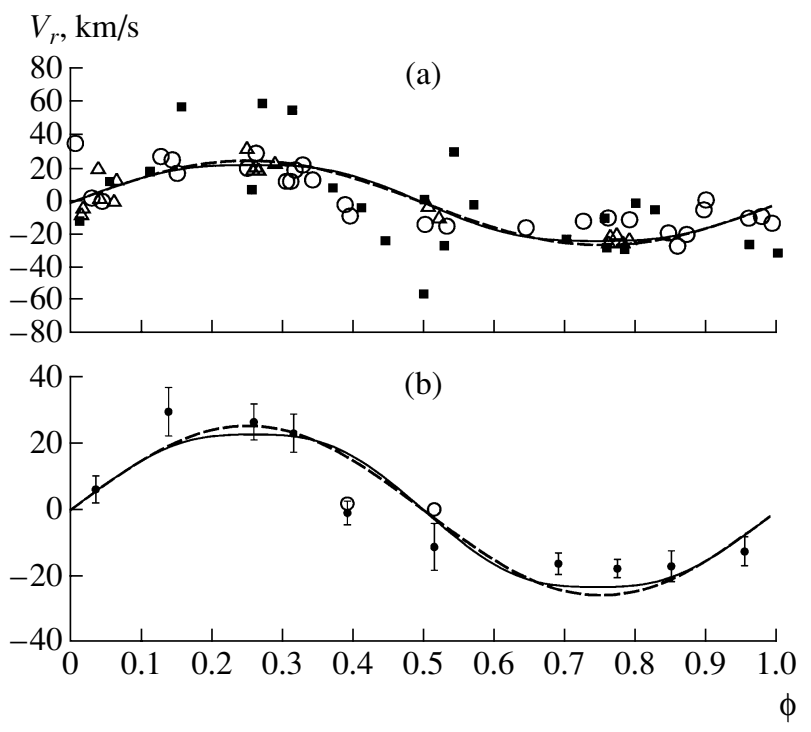

Fig. 4. (a) Master observed radial-velocity curve for the close X-ray binary SMC X-1 (filled squares show spectroscopic data for 1973 from [27]; open circles, spectroscopic data for 1976 from [28]; and open triangles, spectroscopic data for 1989 from [29]). For comparison, theoretical radial-velocity curves are shown for the Roche model (solid curve) and point-mass model (dashed curve), with $m_{x}=1.48 M_{\odot}$ corresponding to the minimum residual for the Roche model computed using method 2. The parameters are collected in Table 3. (b) Radial velocities averaged within phase intervals (filled circles are the mean radial velocities for the phase bins, and open circles, the radial velocities corrected for the normalized anisotropy function of the optical star's wind). For comparison, theoretical radial-velocity curves are shown for the Roche model (solid curve) and point-mass model (dashed curve) with $m_{x}=1.48 M_{\odot}$.

17-18 $M_{\odot}$ and the $\mathrm{X}$-ray pulsar. Its orbital period is 3.89229118 days.

The mean radial-velocity curve (Fig. 4a) was constructed using the data of $[27-29]$. The mean radial velocities measured from the HeI and SiIV absorption lines and the hydrogen Balmer lines are presented in [27]. The radial velocities determined by Hutchings et al. [28] were based on the mean of the shifts of the hydrogen Balmer lines and the HeI, SiIV, and NIII absorption lines in the blue (3900-4000 $)$ ). The radial velocities in [29] were computed using a crosscorrelation method based on the entire spectrum from 4000 to $4395 \AA$ relative to the spectrum of the B3V standard star HR 1174, which has a similar spectral type.

Before plotting the mean curve, we corrected the spectroscopic data of [27-29] for the $\gamma$ velocity. We computed the $\gamma$ velocity by minimizing the sum of squared deviations between the radial velocities that were observed and synthesized in the Roche model 


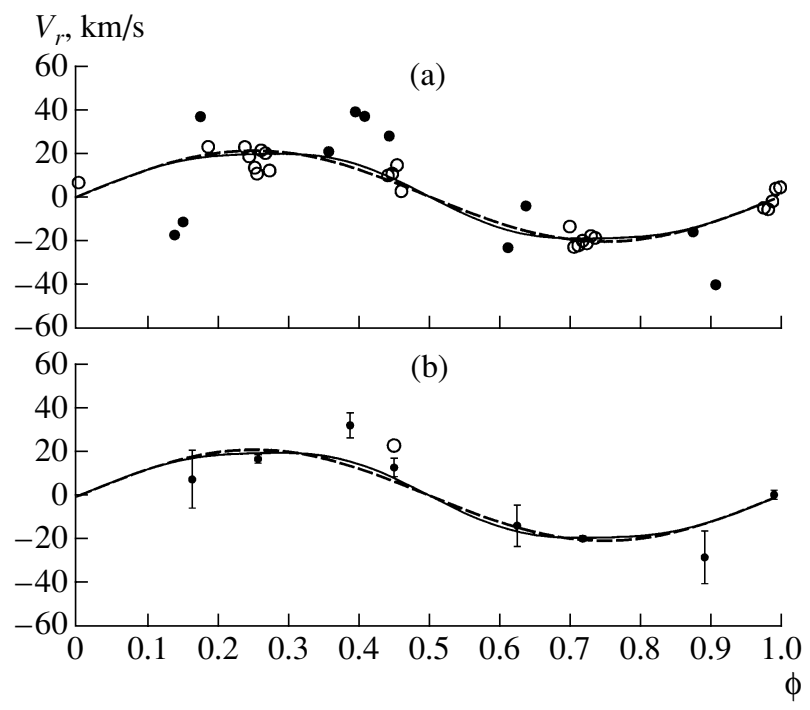

Fig. 5. (a) Master observed radial-velocity curve for the close X-ray binary 4U 1538-52 (filled circles are the spectroscopic data for 1978 of [31], and open circles, the spectroscopic data for 1991 of [32]). For comparison, the theoretical radial-velocity curves are shown for the Roche model (solid curve) and point-mass model (dashed curve), with $m_{x}=1.18 M_{\odot}$ corresponding to the minimum residual in the Roche model computed using method 2. The parameters are collected in Table 3. (b) The radial velocities averaged within phase intervals (filled circles are the mean radial velocities for the phase bins, and open circles, the radial velocities corrected for the normalized anisotropy function of the optical star's wind). For comparison, the theoretical radial velocity curves are shown for the Roche model (solid curve) and point-mass model (dashed curve) with $m_{x}=1.18 M_{\odot}$.

for the same phase. The $\gamma$ velocity enters the algorithm used to synthesize the radial-velocity curves additively, so that the condition that the areas of the radial-velocity curve above and below the $\gamma$ velocity be equal was fulfilled automatically. The $\gamma$ velocities derived from the spectroscopic data of [27-29] were $173.0,181.0$, and $172.7 \mathrm{~km} / \mathrm{s}$.

$4 \mathrm{U}$ 1538-52. This system consists of the X-ray pulsar and a B0Iabe supergiant with a mass of 17$18 M_{\odot}$. The system's orbital period is 3.72844 days. The eccentricity derived from timing of the X-ray pulses is $0.07 \pm 0.09$ [30]. Due to the large uncertainty of this value, we assumed zero eccentricity.

The master radial-velocity curve (Fig. 5a) is based on the spectroscopic data of $[31,32]$. Radial velocities derived from the $\mathrm{H} \beta, \mathrm{H} \gamma$, HeI $4921 \AA$, and HeI $4471 \AA$ absorption lines are presented in [31]. The radial velocities in [32] were computed via the crosscorrelation method using the spectroscopic standard HD 133955 and HeI absorption lines in the range 6290-6710 ̊. These data are in good agreement. We corrected the data from $[31,32]$ for the close binary's

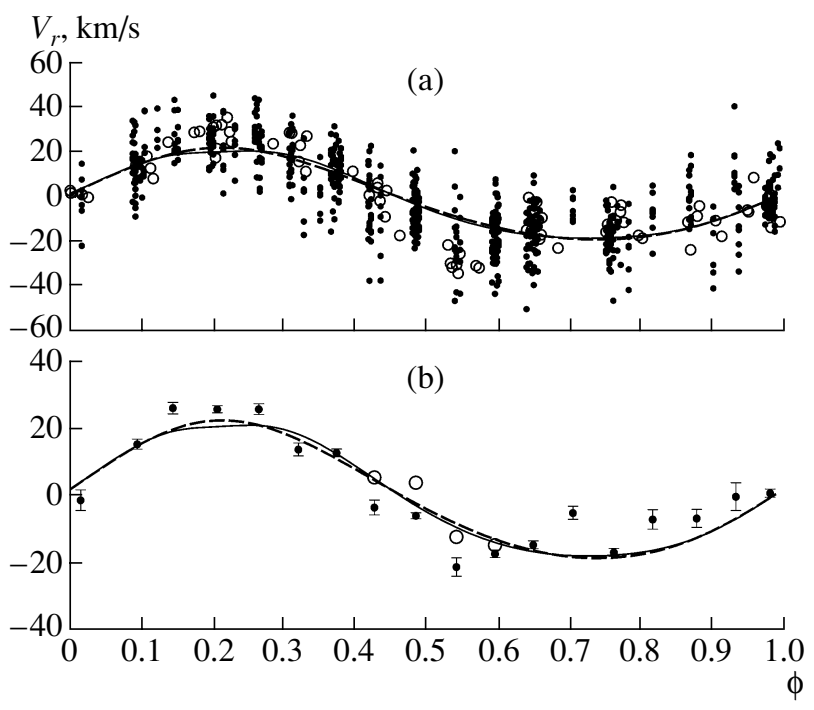

Fig. 6. (a): Master observed radial-velocity curve for the close X-ray binary Vela X-1 (filled circles are the spectroscopic data for 1973 of [33], and open circles, the spectroscopic data for 1995-1996 of [34]). For comparison, the theoretical radial-velocity curves are shown for the Roche model (solid curve) and point-mass model (dashed curve), with $m_{x}=1.93 M_{\odot}$ corresponding to the minimum residual in the Roche model computed using method 2. The parameters are collected in Table 3. (b) The radial velocities averaged within phase intervals (filled circles are the mean radial velocities for the phase bins, and open circles, the radial velocities corrected for the normalized anisotropy function of the optical star's wind). For comparison, the theoretical radial-velocity curves are shown for the Roche model (solid curve) and point-mass model (dashed curve) with $m_{x}=1.93 M_{\odot}$.

$\gamma$ velocity. We derived the $\gamma$ velocities -168.5 and $-146.7 \mathrm{~km} / \mathrm{s}$ using the spectroscopic data of [31, 32], respectively. In this case, the large negative value of the $\gamma$ velocity may reflect radial expansion of the lineformation region near the base of the stellar wind.

Vela $\mathbf{X}-1$. This system contains a B0.5Ibeq supergiant with a mass of 24-25 $M_{\odot}$ and the X-ray pulsar. The components are in an elliptical orbit with $e \simeq 0.0898$, and we adopted this eccentricity. The system's orbital period is 8.964368 days.

The observational material for this system is very rich. We used only the radial velocities of $[33,34]$ for the hydrogen Balmer lines to construct the master radial-velocity curve (Fig. 6a). As a result, data for 1973 and 1995-1996 were included in the master curve; these demonstrated good agreement despite the long time interval separating them.

The Balmer absorption lines from [33] derived from the absolute shifts of the cores of the $\mathrm{H}_{2}-\mathrm{H}_{10}$ absorption lines relative to their laboratory wavelengths indicated increasing stellar-wind velocities with decreasing line number in the series-a Balmer progression $[35,36]$. This effect is due to the negative 
temperature gradient in the star's photosphere, resulting in a dependence of the observed stellar-wind velocity on the excitation potential of the absorption line used to measure this velocity. Thus, the $\mathrm{H} \beta \mathrm{ab}-$ sorption line is formed in higher and, consequently cooler, layers of the stellar photosphere, so that the stellar-wind velocity derived from this line is higher than the stellar-wind velocity measured from Balmer absorption lines with higher numbers.

According to the data of [37], the $\gamma$ velocity derived from the $\mathrm{H} \beta$ line and HeI, NIII, and SiIV ion lines is $-3.5 \pm 0.8 \mathrm{~km} / \mathrm{s}$. The shifts of the lines' cores relative to their laboratory wavelength are due not only to the $\gamma$ velocity but also to the velocity of the stellar wind at its base. If the system's $\gamma$ velocity is much lower than this stellar-wind velocity, it is not possible to determine it from the shifts of the $\mathrm{H}_{2}-\mathrm{H}_{10}$ lines due to the strong stellar wind from the OB supergiant. For example, according to the spectroscopy of [33], the radial velocities derived from the $\mathrm{H}_{2}, \mathrm{H}_{3}, \mathrm{H}_{5}$, and $\mathrm{H}_{6}$ lines are $-29.0,-15.5,-14.0$, and $-8.5 \mathrm{~km} / \mathrm{s}$, respectively. It is evident from these results that the velocity associated with the wind outflow is much higher than the systematic velocity of the center of mass, the latter being $-3.5 \pm 0.8 \mathrm{~km} / \mathrm{s}$ [37]. For this reason, we do not present a $\gamma$ velocity for Vela $X-1$. The radial velocities derived using the $\mathrm{H}_{2}-\mathrm{H}_{10}$ lines from [33] were corrected for the systematic radial velocity (the velocities of the center-of-mass motion and of the stellar wind at its base) individually for each line before including them in the master radialvelocity curve.

The spectral cross-correlation method used in [34] to compute the radial velocities made it unnecessary for us to introduce any further corrections for either the velocity of the stellar wind at its base or the $\gamma$ velocity.

We can see from the above discussion that the radial velocities we used to fit the observational data were derived from both the hydrogen and HeI absorption lines. Therefore, as was mentioned above, we carried out test computations to check the agreement between the theoretical radial-velocity curves based on the $\mathrm{H} \gamma$ and HeI $4471 \AA$ lines (Fig. 1b). The radial velocities for the HeI $4471 \AA$ line were computed using a new version of our algorithm for the synthesis of theoretical absorption-line profiles and of the radial-velocity curves for close $\mathrm{X}$-ray binaries [38]. In contrast to the earlier algorithm, which is described in the cited paper and uses the Balmer profiles from the tables of Kurucz [18] for the local profiles, the new algorithm computes the local profiles for area elements on the stellar surface after constructing the model atmosphere irradiated by the external X-rays. Since the X-ray heating is not strong for most of the

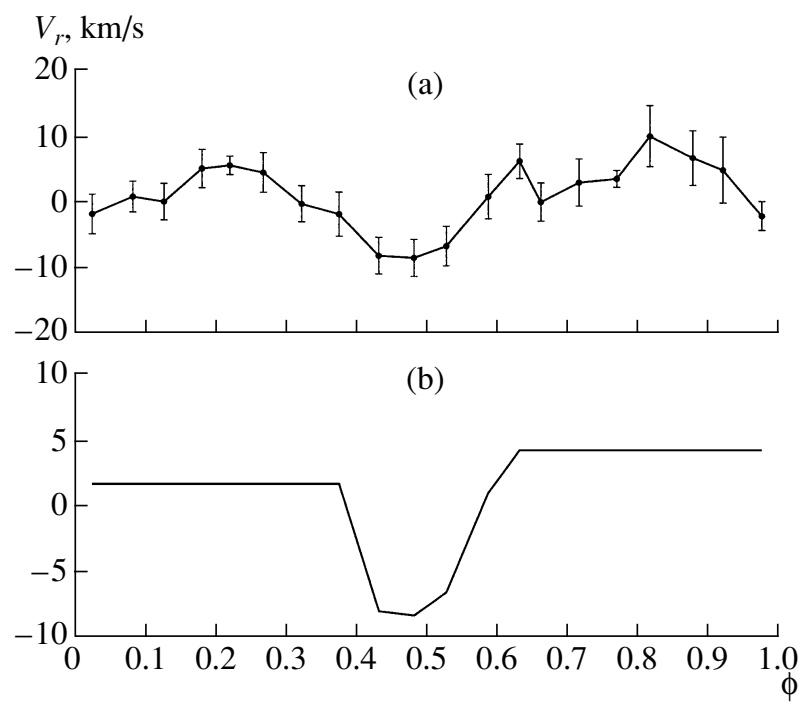

Fig. 7. (a) Anisotropy function of the stellar wind. (b) Same after normalization.

binaries studied here, we deemed it to be admissible to synthesize the theoretical radial-velocity curves using our earlier algorithm, which requires far fewer computer resources. The theoretical radial-velocity curves for the $\mathrm{H} \gamma$ and HeI $4471 \AA$ lines shown in Fig. $1 \mathrm{~b}$ demonstrate good agreement, and, as was already mentioned, we used the theoretical radialvelocity curves based on the $\mathrm{H} \gamma$ line for our analysis of the master set of observational data for the Balmer lines and HeI absorption lines.

\section{THE EFFECT OF ANISOTROPY OF THE STELLAR WIND \\ IN THE ATMOSPHERE OF THE OB STAR}

Early-type stars posses high mass-loss rates, up to $10^{-7}-10^{-5} \dot{M}_{\odot} /$ year. The outflow velocity in the zone in which the absorption lines are formed can be as high as $\sim 10-20 \mathrm{~km} / \mathrm{s}$, and, thus, becomes comparable to the orbital velocity of the OB giant in the system.

The isotropy of the stellar wind in a close binary is disrupted by the gravitational action of the compact object. The optical star is already in a nonuniform gravitational field. The wind velocity near the Lagrangian point $\mathrm{L}_{1}$ increases; this is observed (Figs. 2a, 3a, 4a, 5a, 6a) as an excess negative radial velocity (toward the observer) at phase 0.5 , when the $\mathrm{X}$-ray source is in front of the OB star.

This strongly hinders correct interpretation of the radial-velocity curve for close binaries with earlytype optical components. The anisotropy of the stellar wind is a source of errors when searching for a system's spectroscopic orbital elements [14], and 
this anisotropy must likewise be taken into account when fitting the radial-velocity curve of the optical component of a close binary. Bearing in mind the difficulties of constructing theoretical models for an anisotropic wind from a distorted star, we approached this problem empirically.

Before fitting the radial-velocity curves of the systems, we calculated the phase dependences of the differences $V_{o b s}-V_{\text {teor }}$ for each system (with the exception of $4 U$ 1538-52, due to the low number of data points and their nonuniform distribution in orbital phase); $V_{o b s}$ is the observed radial velocity for a given orbital phase and $V_{\text {teor }}$ is the theoretical value in the Roche model without taking into account the wind. Jumping somewhat ahead, we note that we used the mass of the relativistic object computed using the second method (i.e., not taking into account the radial velocities observed at phases from 0.4 to 0.6 ) when computing $V_{\text {teor }}$. Such mass estimates for X-ray pulsars are most reliable.

The resulting radial-velocity deviations for all four systems were combined and averaged within phase bins with a width of 0.05 . Since the structure of the spectroscopic data for Vela X-1 was such that radial velocities derived from the $\mathrm{H}_{2}-\mathrm{H}_{10}$ absorption lines fell at the same phase, we first averaged these within each orbital phase. We adopted the mean velocity from all the absorption lines for $V_{o b s}$ (i.e., from $\left.\mathrm{H}_{2}-\mathrm{H}_{10}\right)$ at a given epoch. Unaveraged data were used for the other systems.

The mean curve of the radial-velocity deviations, $V_{o b s}-V_{t e o r}$, for the four systems is presented in Fig. 7a. We will call this relation the stellar-wind anisotropy function. The relation in Fig. 7a clearly demonstrates an excess negative radial velocity (toward the observer) at phases from 0.4 to 0.6 , when the nose of the star is turned toward the observer. This result is in qualitative agreement with the physics of anisotropic stellar-wind outflows.

The use of the stellar-wind anisotropy function to correct the observed radial velocities is justified by the fact that it is essentially an empirical relation. We normalized the relation for this purpose. The $V_{o b s}-$ $V_{\text {teor }}$ residuals were averaged for the phase intervals $0-0.4$ and $0.6-1.0$. The resulting mean values, 1.79 and $4.32 \mathrm{~km} / \mathrm{s}$, were adopted as the zero points for correcting the observed radial velocities in the phase intervals $0.0-0.5$ and $0.5-1.0$, respectively. The normalized empirical stellar-wind anisotropy function is shown in Fig. 7b.

\section{INTERPRETATION \\ OF THE RADIAL-VELOCITY CURVES}

Our aim was to determine the mass of the $\mathrm{X}$-ray pulsars from the master radial-velocity curves in the
Roche model, taking into account the anisotropy of the stellar wind from the OB star.

We applied a simple method with an exhaustive parameter search and found multiple solutions varying the desired parameter. In our case, the unknown was $m_{x}$ and the mass of the compact object in the close X-ray binary was varied during the solutions, with all other parameters of the close binary being fixed. The values of these fixed parameters were derived from analyses of the photometric light curves, $\mathrm{X}$-ray eclipse data, and timing of the X-ray pulses. The close-binary parameters used to synthesize the radial-velocity curves are presented in Table 3.

It is often very difficult to measure the true radial velocity of the optical star. Complex processes on the surface of the supergiant and in gaseous structures in the system can lead to both systematic and random deviations. We were faced with the question of how to best take into account the systematic errors. In addition to the effect of anisotropy in the optical star's wind, tidal gravitational waves on the stellar surface could be a source of systematic errors if the orbit is elliptical. The recent study [40] demonstrated that there was no correlation between the orbital phase and the phase of the gravitational tidal waves. Consequently, tidal waves on the optical star's surface are a source of random errors and can be reduced by averaging the data from many nights of observations.

In addition to gravitational-tidal perturbations of the OB supergiant's surface, additional absorption and photoionization effects [41] in the complex gaseous structures in the close binary can be sources of random errors in the observed radial velocities.

To reduce the influence of random errors due to various effects, we averaged the radial velocities within phase intervals. Our subsequent analysis of the radial-velocity curves was based on these mean observed radial velocities. The averaged radialvelocity curves for the program binaries are presented in Figs. 2b, 3b, 4b, 5b, and 6b.

We tested various hypotheses using the Fisher ratio test, which takes into account the most complete information on the averaged data. We describe our adaptation of the Fisher ratio test for the analysis of the radial-velocity curves below. Let $\bar{V}_{j}{ }^{\text {obs }}$ be the observed mean radial velocity for the phase interval centered at phase $\bar{\phi}_{j} ; V_{j}^{\text {teor }}$, the theoretical radial velocity value at this phase; and $\sigma_{j}$, the rms deviation of $\bar{V}_{j}^{\text {obs }}$ from the radial velocities observed in the given phase interval centered at $\bar{\phi}_{j}$. Let $M$ be the number of the phase intervals, and $n_{j}$, the number of radial 
Table 3. Numerical parameters used to synthesize the radial-velocity curves of the close X-ray binaries in the Roche model

\begin{tabular}{l|c|c|c|c|c}
\hline Parameters & Cen X-3 & LMC X-4 & SMC X-1 & 4U 1538-52 & Vela X-1 \\
\hline$P$, days & 2.0871390 & 1.40839 & 3.89229118 & 3.72844 & 8.964368 \\
$e$ & 0.0 & 0.0 & 0.0 & 0.0 & 0.0898 \\
$\omega$, deg* & 0.0 & 0.0 & 0.0 & 0.0 & 332.59 \\
$i$, deg & 82 & 65 & 65 & 65 & 73 \\
$\mu^{* *}$ & 0.99 & 0.99 & 0.97 & 0.95 & 0.95 и 0.99 \\
$f$ & 0.95 & 0.65 & 0.95 & 0.94 & 0.69 \\
$T_{\text {eff }}, \mathrm{K}$ & 35000 & 35000 & 25000 & 25000 & 25000 \\
$\beta$ & 0.25 & 0.25 & 0.25 & 0.25 & 0.25 \\
$k_{x}$ & 0.05 & 1.4 & 0.25 & 0.0025 & 0.003 \\
$A$ & 0.5 & 0.5 & 0.5 & 0.5 & 0.5 \\
$u^{* * *}$ & 0.30 & 0.30 & 0.38 & 0.38 & 0.38 \\
$K_{x}, \mathrm{~km} / \mathrm{s}$ & 414.1 & 400.6 & 301.5 & 309.0 & 278.1 \\
\hline
\end{tabular}

${ }^{*}$ The longitude of the periastron of the optical component is given.

** The value for Vela X-1 corresponds to the orbit periastron.

*** Data of [39].

velocities averaged in a given phase interval. We can calculate the residual as follows:

$$
\Delta\left(m_{x}\right)=\frac{\sum_{j=1}^{M}\left(n_{j}-1\right)}{M} \frac{\sum_{j=1}^{M} n_{j}\left(V_{j}^{\text {teor }}-\bar{V}_{j}^{\text {obs }}\right)^{2}}{\sum_{j=1}^{M} n_{j}\left(n_{j}-1\right) \sigma_{j}^{2}} .
$$

The variable $\Delta\left(m_{x}\right)$ will be distributed according to a Fisher law, $F_{M, \sum_{j=1}^{M}\left(n_{j}-1\right), \alpha}$ [42]. Let us adopt the significance level $\alpha$. The confidence set for the desired parameter, $m_{x}$, will then consist of the values satisfying the condition

$$
\Delta\left(m_{x}\right) \leq F{ }_{M, \sum_{j=1}^{M}\left(n_{j}-1\right), \alpha} .
$$

We solved the inverse problem using the Roche and point-mass models. The latter was used only to reveal disagreements between the results for the two models. We analyzed the mean radial-velocity curves for each system using three methods.

Method 1. Using all the mean observed radial velocities, with no correction for anisotropy of the stellar wind.

Method 2. Rejecting mean observed radial velocities at phases $0.4-0.6$ that are most strongly distorted by the effects of the stellar-wind anisotropy.
Method 3. Using the mean radial velocities at phases $0.4-0.6$ corrected for the stellar-wind anisotropy function.

Let us consider the details of our procedures for determining the compact object's mass using the first system, Cen X-3, as an example.

Cen X-3. The mean radial-velocity curve for Cen X-3 had 79 data points. Despite the fact that the points were uniformly distributed in orbital phase (Fig. 2a), we can identify close groups of points with similar orbital phases. In the case of Cen X-3, we isolated 11 such groups, within which we averaged the observed radial velocities. The radial velocities averaged within the phase bins are shown in Fig. $2 \mathrm{~b}$.

The mean radial velocities show the strongest deviations from the standard curve at phases 0.4-0.6. The supergiant's excess negative radial velocity at these phases is due to the anisotropy of the stellar wind.

Recall that we determined the unknown $m_{x}$ by carrying out an exhaustive search among its possible values. In the process, we varied the mass of the relativistic component so that the semiamplitude of its radial-velocity curve remained unchanged, $K_{x}=$ $414.1 \mathrm{~km} / \mathrm{s}$ [43]. For more detail on the method used to compute the optical component's mass by running through possible masses for the relativistic component, see the Appendix. The remaining binary parameters (Table 3 ) were fixed. 

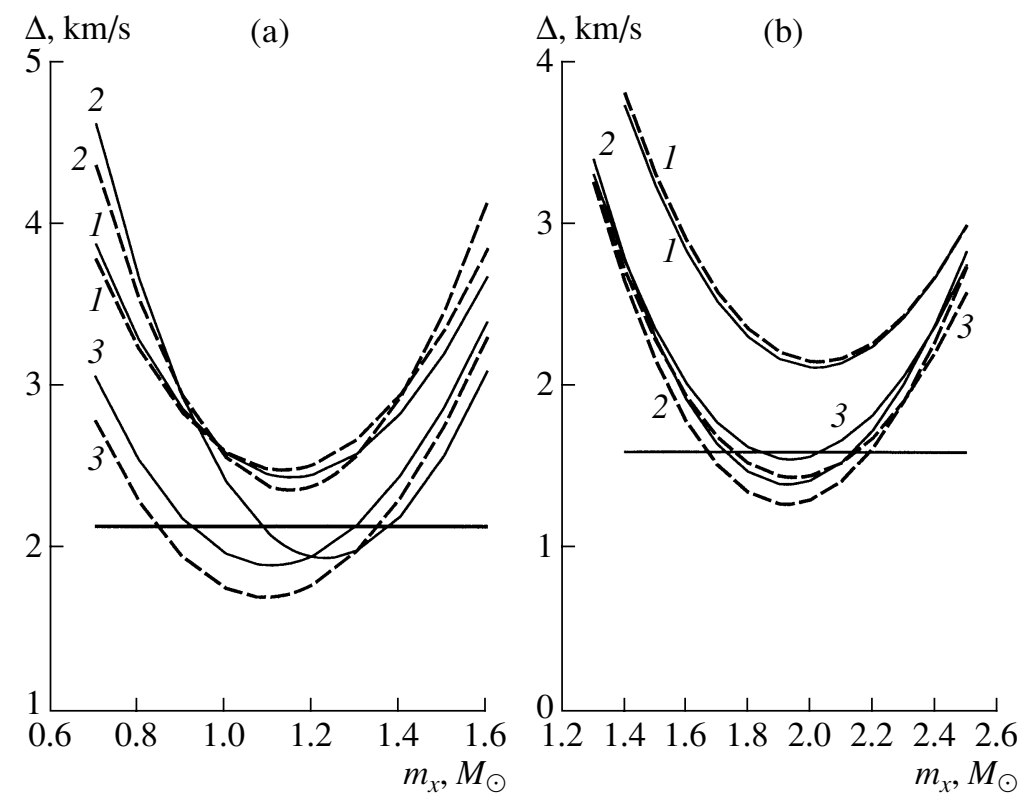

Fig. 8. Deviations of the observed mean radial-velocity curves for (a) Cen X-3 and (b) Vela X-1 from the synthetic curves in the Roche model (solid curve) and point-mass model (dashed curve) obtained using methods 1,2 , and 3 . The method used to derive the residuals is indicated by a number near the curve. The horizontal lines correspond to the critical levels in the Fisher ratio test, $\Delta_{8,47}=2.14$ (a) and $\Delta_{18,782}=1.605$ (b), for a significance level of $5 \%$.

The residual yielded by analyzing the observed radial velocities using method 1 is shown in Fig. 8a. The significance level adopted for the computations was $5 \%$. We can see in this figure that the model without allowance for the wind anisotropy must be rejected at this significance level. The $m_{x}$ value corresponding to the minimum residual is presented in Table 4 .

Interpretation of the radial-velocity curve is hindered by the strong deviation of the data points from their computed positions near phase 0.5. This does not fit into the Roche model with an isotropic stellar wind. We therefore excluded in the next stage observed radial velocities at phases $0.4-0.6$ during the computations, and our analysis of the fitted curves did not take them into account (method 2). The behavior of the residuals is shown in Fig. 8a. We can see that the point-mass model must be rejected according to the Fisher test, whereas the Roche model, which is more adequate for describing the physics of processes in close binaries, is acceptable. We accordingly adopt the Roche model, not because it is necessarily correct, but because there is no reason to reject it (see the review [44] for details).

The mass of the $\mathrm{X}$-ray pulsar corresponding to the minimum residual in the Roche model is $1.63 M_{\odot}$. The minimum residual for the point-mass model is reached for $1.47 M_{\odot}$. This large a discrepancy can be explained by the difference in the shapes of the synthetic curves for the Roche and point-mass models (Fig. 1a). The barycenter of the system is inside the body of the optical star $(q<1)$, so that some fraction of the optical star's emitting surface is moving in the same direction as the relativistic component. This leads to a lower amplitude of the synthesized radial-velocity curve in the Roche model, all the other parameters being the same, and, hence, to a higher mass for the relativistic object.

In the computations with method 3 , we corrected the mean radial velocities at phases $0.4-0.6$ for the mean normalized wind anisotropy function. This correction makes the deviation between the synthetic and observed radial velocity values much smaller (Fig. 2b). The differences between the theoretical and corrected observed radial-velocity curves are presented in Fig. 8a. We can see that, in this case, the Fisher ratio test indicates both the Roche and point-mass models to be acceptable.

The resulting masses, along with the masses of the $\mathrm{X}$-ray pulsars in the other systems, are collected in Table 4.

LMC X-4. The master radial-velocity curve contains about 70 data points (Fig. 3a); the averaged master radial-velocity curve is presented in Fig. 3b. The mean velocities in the phase bins were formed using from three to ten points. These mean observed velocities are in good agreement with the theoretical values.

The residuals obtained for the Roche and pointmass models using methods 1 and 2 indicate that both models are acceptable according to the Fisher 
Table 4. Masses of X-ray pulsars in the close binaries with supergiants derived from fitting the mean observed radialvelocity curves in the Roche and point-mass models (the confidence interval is given for the $95 \%$ confidence level)

\begin{tabular}{l|c|c|c|c|c|c}
\hline \multirow{2}{*}{ Name } & \multicolumn{3}{|c|}{ Roche model } & \multicolumn{3}{c}{ Point-mass model } \\
\cline { 2 - 7 } & Method 1 & Method 2 & Method 3 & Method 1 & Method 2 & Method 3 \\
\hline Cen X-3 & 1.14 & $1.22_{-0.14}^{+0.15}$ & $1.10_{-0.05}^{+0.05}$ & 1.12 & 1.14 & $1.09_{-0.18}^{+0.18}$ \\
LMC X-4 & $1.55_{-0.27}^{+0.29}$ & $1.63_{-0.47}^{+0.42}$ & 1.60 & $1.40_{-0.29}^{+0.30}$ & $1.47_{-0.43}^{+0.47}$ & $1.43_{-0.05}^{+0.08}$ \\
SMC X-1 & $1.40_{-0.29}^{+0.33}$ & $1.48_{-0.42}^{+0.47}$ & $1.40_{-0.45}^{+0.49}$ & $1.30_{-0.31}^{+0.33}$ & $1.36_{-0.39}^{+0.41}$ & $1.30_{-0.45}^{+0.49}$ \\
4U 1538-52 & $1.21_{-0.26}^{+0.28}$ & $1.18_{-0.27}^{+0.29}$ & 1.26 & 1.16 & $1.13_{-0.11}^{+0.11}$ & 1.21 \\
Vela X-1 $(\mu=0.95)^{*}$ & 2.02 & $1.93_{-0.24}^{+0.25}$ & $1.94_{-0.10}^{+0.08}$ & 2.02 & $1.92_{-0.28}^{+0.30}$ & $1.94_{-0.18}^{+0.21}$ \\
Vela X-1 $(\mu=0.99)^{*}$ & 2.02 & $1.93_{-0.21}^{+0.19}$ & 1.94 & 2.02 & $1.92_{-0.28}^{+0.30}$ & $1.94_{-0.18}^{+0.21}$ \\
\hline
\end{tabular}

* The confidence interval is given for the error of the mean artificially increased twofold.

test. Because of the initial good agreement of the mean observed and theoretical radial velocities, the residual increases when the observed radial-velocity curve is analyzed using method 3 . The increase in the difference between the theoretical and observed radial velocities is quite apparent in Fig. 3b. When the observed mean radial-velocity curve is analyzed using method 3, the point-mass model is rejected by the Fisher test, whereas the Roche model is acceptable. The resulting masses and confidence intervals are presented in Table 4.

SMC X-1. The mean curve contained 70 data points (Fig. 3a). The averaged radial velocities are presented in Fig. 4b; the observed radial velocities at phases 0.40 and 0.51 deviate fairly strongly from the theoretical values.

The analysis of the mean observed radial-velocity curves using any of the three methods indicates that both the Roche and point-mass models are acceptable according to the Fisher ratio test. Correction of the mean observed radial velocities at phases 0.40 and 0.51 reduces the disagreement between the synthetic and observed radial velocities (Fig. 4b); i.e., the residuals obtained using method 3 are lower than those for method 1 . This is reflected in the broader confidence interval for the X-ray pulsar masses satisfying the Fisher test at the $95 \%$ confidence level for method 3 compared to method 1 (Table 4).

$4 U$ 1538-52. The master radial-velocity curve contains 36 data points (Fig. 5a); the values averaged within each group are presented in Fig. 5b. Because of the sparseness of the spectroscopic data, the averaging in some phase intervals was based on radial velocities measured on a single night, making it impossible to reduce the influence of random errors. This could be the origin of the excess positive velocity at phase 0.45 .

When analyzing the mean observed radial velocities using method 1 , the Roche model passes the
Fisher ratio test, whereas the point-mass model is rejected. With the radial velocity at phase 0.45 excluded (model 2), both the Roche and point-mass models pass the Fisher test. Since an excess positive radial velocity is observed at phase 0.45 , the correction for the normalized stellar-wind anisotropy function only increases the discrepancy between the mean observed and theoretical radial velocities. Thus, the increased residual yielded by method 3 results in both models being rejected by the Fisher test. The resulting masses and confidence intervals are presented in Table 4.

Vela $\mathbf{X}-1$. The master radial-velocity curve included 782 data points subdivided into 18 groups (Fig. 6a). The values averaged within each group are shown in Fig. 6b. Each phase bin used for the averaging contains many ( 28 to 82 ) data points, leading to low standard deviations. Thus, the residuals obtained using method 1 , method 2 , and method 3 are $8.59,6.00$, and 6.59 , respectively, with the quantiles being $1.69,1.605$, and 1.69. The low uncertainties in the averaged data impose stringent requirements on the models, so that even the Roche model with the stellar-wind anisotropy taken into account is rejected by the Fisher test, testifying to the complexity of the physics of the absorption-line-formation processes.

Artificially increasing the standard deviations, $\sigma_{j}$, by a factor of 1.5 reduced the residuals for method 2 to 1.92 , whereas the critical level for the Fisher ratio test with a $5 \%$ significance level is 1.605 . The difference between the critical level for the Fisher test with the $5 \%$ significance level and the residuals derived using the other methods using $1.5 \sigma_{j}$ are even higher. When the standard deviations, $\sigma_{j}$, in (1) were artificially doubled, the models for methods 2 and 3 were deemed acceptable by the Fisher test. The model for method 1 is rejected even after doubling the standard 
deviations and analyzing the full, uncorrected radialvelocity curve.

The above findings indicate that the Roche model is not adequate to the high-accuracy radial-velocity curve of the optical star in the Vela X-1 system, making the mass of the $\mathrm{X}$-ray pulsar, $m_{x}$, derived for this system relatively uncertain.

Because of the large uncertainty in the Roche-lobe filling coefficient, $\mu$, we carried out computations for $\mu=0.95$ and 0.99 , keeping the remaining conditions the same (this corresponds to two lines in Table 4). The deviation of the mean observed radial-velocity curve from the synthetic curve for the Roche model is much lower for $\mu=0.95$ than for $\mu=0.99$, which is reflected in a wider confidence interval for the better fit (Table 4). However, the mass of the X-ray pulsar derived from the residual minimum remains the same for the two cases. We conclude that the radial-velocity curve is insensitive to relatively small variations of the Roche-lobe filling coefficient.

Figure $8 \mathrm{~b}$ shows the behavior of the residuals (for $\mu=0.95$ ) found using each of the methods. Table 4 presents the $\mathrm{X}$-ray pulsar masses corresponding to the minimum residuals for models rejected by the Fisher ratio test. It is not possible to derive confidence intervals in these cases.

\section{CONCLUSIONS}

This paper has presented estimates of the masses of $\mathrm{X}$-ray pulsars that are most adequate to the entire set of observational data for $\mathrm{X}$-ray binaries with $\mathrm{OB}$ supergiants. In contrast to earlier studies, we have considered not only the semiamplitude of the radialvelocity curve but also its overall shape.

Our investigation demonstrates that a pure Roche model is insufficient for analyzing the radial-velocity curves of X-ray close binaries with early-type optical stars. This model is often rejected by the Fisher ratio test due to the presence of an excess negative radial velocity near phase 0.5 . In a number of cases, only taking into account the wind anisotropy makes it possible to accept the model based on the Fisher test, thereby enabling us to obtain reliable estimates of the neutron-star masses in the X-ray binaries and their confidence intervals.

This study has yielded masses of the X-ray pulsars derived in the Roche model, with the stellar-wind anisotropy taken into account empirically. This model is much more realistic than earlier models based on point masses in Keplerian orbits. Note that the mass estimates for X-ray pulsars in binaries with supergiants obtained in the point-mass models of [13] are in good agreement with our own determinations using the same model with two point masses.
Let us now consider the X-ray pulsar mass estimates for the Roche model. They are systematically higher than the masses derived in the pointmass model, by on average $\sim 10 \%$ ( Table 4 ). Our test computations for the SMC X-1 system demonstrated that the systematic excess of the X-ray pulsar masses obtained for the Roche model did not depend on the orbital inclination $i$ (see the Appendix). Thus, we conclude that the $\mathrm{X}$-ray pulsar masses were systematically too low (by 5-10\%) in all earlier studies based on point-mass models.

The masses of the $\mathrm{X}$-ray pulsars in LMC X-4 and SMC X-1, $1.63_{-0.47}^{+0.42} M_{\odot}$ and $1.48_{-0.42}^{+0.47} M_{\odot}$, respectively, are somewhat higher than the standard mass for a neutron star, $1.35 \pm 0.04 M_{\odot}$ [21], though they can be reconciled with a mass of $1.35 M_{\odot}$ within our confidence intervals. It is difficult to explain the excess of the X-ray pulsar masses over the standard value as being due to the accumulation of accreted matter from an accretion disk onto the neutron star's surface. A mass of about $0.01 M_{\odot}$ could settle onto the pulsar's surface over the optical component's life time during the stage when the Roche lobe is close to being filled, $\sim 10^{5}$ years, if the mass-loss rate of the optical star is $10^{-7}-10^{-6} \dot{M}_{\odot} /$ year, taking into account the size of the close binary and the relativistic star's gravitational capture radius.

The X-ray pulsar masses in the Cen X-3, LMC $\mathrm{X}-4$, SMC X-1, and 4U 1538-52 systems derived using the most reliable method, in the Roche model excluding the mean radial velocities near phase 0.5 , are $1.22_{-0.14}^{+0.15} \quad M_{\odot}, 1.63_{-0.47}^{+0.42} M_{\odot}, 1.48_{-0.42}^{+0.47} \quad M_{\odot}$, and $1.18_{-0.27}^{+0.29} M_{\odot}$, respectively. The mean of the four X-ray pulsar masses is $1.37 \pm 0.15 M_{\odot}$ at the $95 \%$ confidence level (the mass of the pulsar in the Vela X-1 system was not used for the average due to its anomalously high value). This mean X-ray pulsar mass agrees with the standard radio pulsar mass, $1.35 \pm 0.04 M_{\odot}$, within the errors [21].

Our test computations in the Roche model for various orbital inclinations, $i$, demonstrated the validity of the relation $m_{x} \sim \sin ^{-3} i$ (see the Appendix for more details). Thus, the X-ray pulsar masses derived in the Roche model can easily be recalculated if their orbital inclinations are refined.

An important result of this study is the mass estimate for the compact object in the Vela X-1 X-ray binary. The earlier estimate was $1.86_{-0.32}^{+0.32} M_{\odot}$ at the 95\% significance level [34], considerably in excess of the standard value, $1.35 \pm 0.04 M_{\odot}$. Our analysis of the radial-velocity curve in the Roche model taking into account the wind anisotropy slightly increased the mass of the $\mathrm{X}$-ray pulsar in this system, to $1.93_{-0.21}^{+0.19} M_{\odot}$ at the $95 \%$ confidence level. Note 
Table 5. Masses of the SMC X-1 pulsar derived using the Roche and point-mass model for various orbital inclinations (confidence intervals are given for the $95 \%$ confidence level)

\begin{tabular}{l|c|c|c|c|c|c}
\hline \multirow{2}{*}{ Orbital inclination } & \multicolumn{3}{|c|}{ Roche model } & \multicolumn{3}{c}{ Point-mass model } \\
\cline { 2 - 7 } & Method 1 & Method 2 & Method 3 & Method 1 & Method 2 & Method 3 \\
\hline$i=65^{\circ}$ & $1.40_{-0.29}^{+0.33}$ & $1.48_{-0.29}^{+0.33}$ & $1.40_{-0.45}^{+0.49}$ & $1.30_{-0.31}^{+0.33}$ & $1.36_{-0.39}^{+0.41}$ & $1.30_{-0.45}^{+0.49}$ \\
$i=75^{\circ}$ (case A) & $1.15_{-0.24}^{+0.27}$ & $1.22_{-0.36}^{+0.37}$ & $1.15_{-0.37}^{+0.40}$ & $1.08_{-0.25}^{+0.27}$ & $1.12_{-0.31}^{+0.35}$ & $1.43_{-0.38}^{+0.41}$ \\
$i=75^{\circ}$ (case B) & 1.156 & 1.223 & 1.156 & 1.074 & 1.123 & 1.074 \\
$i=85^{\circ}$ (case A) & $1.05_{-0.22}^{+0.24}$ & $1.11_{-0.32}^{+0.34}$ & $1.05_{-0.33}^{+0.37}$ & $0.98_{-0.24}^{+0.24}$ & $1.02_{-0.28}^{+0.32}$ & $0.98_{-0.33}^{+0.38}$ \\
$i=85^{\circ}$ (case B) & 1.054 & 1.114 & 1.054 & 0.978 & 1.024 & 0.978 \\
\hline
\end{tabular}

again that our Roche model for the Vela X-1 system was rejected by the Fisher ratio test, so that the X-ray pulsar's mass, $1.93 M_{\odot}$, cannot be considered very trustworthy. A "hard" equation of state must be used to explain the existence of neutron stars with masses this high. Studies in this direction are very promising.

\section{ACKNOWLEDGMENTS}

This study was supported by the Russian Foundation for Basic Research (project code 02-02-17524) and a grant from the program "Leading Scientific Schools of Russia." We thank V.V. Shimanskil for helpful advice.

APPENDIX

When solving the inverse problem, we varied the mass of the X-ray pulsar, $m_{x}$, so that the semiamplitude of its radial-velocity curve, $K_{x}$, remained unchanged, since this parameter is known to high accuracy from X-ray timing data (Table 2). To keep $K_{x}$ constant as the mass of the relativistic component, $m_{x}$, varied, we had to vary the mass of the optical star, $m_{v}$, as well. We used the classical formulas to relate the mass of the optical component, $m_{v}$, to each value of the X-ray pulsar's mass, $m_{x}$ :

$$
\begin{aligned}
m_{x} \sin ^{3} i & =1.038 \times 10^{-7} P\left(1-e^{2}\right)^{3 / 2} \\
& \times K_{v}\left(K_{x}+K_{v}\right)^{2} \\
m_{v} \sin ^{3} i & =1.038 \times 10^{-7} P\left(1-e^{2}\right)^{3 / 2} \\
& \times K_{x}\left(K_{v}+K_{x}\right)^{2} .
\end{aligned}
$$

The point-object model is quite applicable to the $\mathrm{X}$-ray pulsar, justifying the use of (2). The value of $K_{v}$ it gives for fixed $m_{x}$ and $i$ characterizes the true velocity of the optical star's center of mass. Due to the various reasons mentioned above (the pear-like shape of the stars, anisotropic winds, heating effects, etc.), the observed $K_{v}$ can differ from the value of $K_{v}$ found from (2). Substituting the true $K_{v}$ derived from (2) into (3), we find the mass of the optical star, $m_{v}$, for the specified value of $i$. In this manner, relating the mass of the optical component to that of the relativistic component, we can keep the semiamplitude of the relativistic component's radial-velocity curve, $K_{x}$, constant. Note also that (3) contains only a quadratic dependence on $K_{v}$, whereas the dependence on $K_{x}$ is cubic. This is reflected in the fact that varying $m_{x}$ by a factor of 2.7 leads to a change in the optical component's mass by only a factor of 1.14 .

In the model with two point masses, the masses of the optical star, $m_{v}$, and of the X-ray pulsar, $m_{x}$, depend on the orbital inclination, $i$, as $m_{v} \sim \sin ^{-3} i$ and $m_{x} \sim \sin ^{-3} i$. We should check if the relation $m_{x} \sim \sin ^{-3} i$ is valid when the $X$-ray pulsar's mass is determined using the Roche model for the optical star from the minimum difference between the observed mean radial-velocity curve and the curve synthesized in the Roche model.

For this purpose, we also analyzed the mean radial-velocity curve of the SMC X-1 system in the Roche model using the three methods for orbital inclinations of $75^{\circ}$ and $85^{\circ}$. The result is presented in Table 5, where it is called "case A." Having the mass obtained earlier in the Roche model for an orbital inclination of $65^{\circ}$ (Table 4 ), we recalculated this mass for the orbital inclinations $75^{\circ}$ and $85^{\circ}$ according to the formulas

$$
\begin{aligned}
& m_{x}\left(75^{\circ}\right)=m_{x}\left(65^{\circ}\right) \frac{\sin ^{3} 65^{\circ}}{\sin ^{3} 75^{\circ}}, \\
& m_{x}\left(85^{\circ}\right)=m_{x}\left(65^{\circ}\right) \frac{\sin ^{3} 65^{\circ}}{\sin ^{3} 85^{\circ}},
\end{aligned}
$$

where $m_{x}\left(65^{\circ}\right), m_{x}\left(75^{\circ}\right)$, and $m_{x}\left(85^{\circ}\right)$ are the masses of the X-ray pulsar for orbital inclinations of $65^{\circ}$, $75^{\circ}$, and $85^{\circ}$. The results are presented in Table 5, where they are called "case B." We can see that the masses $m_{x}$ derived from the minimum residual in the Roche model for the orbital inclinations $75^{\circ}$ and $85^{\circ}$ 
(case A) are very close to the masses $m_{x}$ obtained using (4) and (5) (case B). Thus, the masses $m_{x}$ derived in the Roche model for the optical star (Table 4) can easily be recalculated using the formula $m_{x} \sim \sin ^{-3} i$ if the orbital inclination, $i$, is refined.

\section{REFERENCES}

1. L. D. Landau, Phys. Z. Sowjetunion 1, 285 (1932).

2. W. Forman et al., Astrophys. J., Suppl. Ser. 38, 357 (1978).

3. Ya. B. Zel'dovich, Dokl. Akad. Nauk SSSR 155, 67 (1964)

4. E. E. Salpeter, Astrophys. J. 140, 796 (1964).

5. N. I. Shakura and R. A. Sunyaev, Astron. Astrophys. 24, 337 (1973).

6. J. E. Pringle and M. J. Rees, Astron. Astrophys. 21, 1 (1972).

7. I. D. Novikov and K. S. Thorne, in Black Holes, Ed. by C. De Witt and B. S. De Witt (Gordon and Breach, London, 1973), p. 343.

8. S. L. Shapiro and S. A. Teukolsky, Black Holes, White Dwarfs, and Neutrons Stars: The Physics of Compact Objects (Wiley, New York, 1983).

9. W. D. Arnett and R. L. Bowers, Astrophys. J., Suppl. Ser. 33, 415 (1977).

10. B. Datta, Fundam. Cosmic Phys. 12, 151 (1988).

11. R. Stock, Nature 337, 319 (1989).

12. G. E. Brown and H. A. Bethe, Astrophys. J. 423, 659 (1994).

13. M. H. van Kerkwijk, J. van Paradijs, and E. J. Zuiderwijk, Astron. Astrophys. 303, 497 (1995).

14. M. Milgrom, Astron. Astrophys. 70, 763 (1978).

15. E. A. Antokhina and A. M. Cherepashchuk, Astron. Zh. 71, 420 (1994) [Astron. Rep. 38, 367 (1994)].

16. E. A. Antokhina, Astron. Zh. 73, 532 (1996) [Astron. Rep. 40, 483 (1996)].

17. R. E. Wilson, Astrophys. J. 234, 1054 (1979).

18. R. L. Kurucz, Astrophys. J., Suppl. Ser. 40, 1 (1979).

19. A. M. Cherepashchuk, N. A. Katysheva, Khruzina, and C. Yu. Shugarov, Highly Evolved Close Binary Stars: Catalog (Netherland Gordon and Breach Sci. Publ., 1996), Vol. 1, Part 1, p. 82.

20. A. V. Goncharskiı̆, S. Yu. Romanov, and A. M. Cherepashchuk, Finite-Parametric Inverse Astrophysical Problems (Mosk. Gos. Univ., Moscow, 1991), p. 105 [in Russian].

21. S. E. Thorsett and D. Chakrabarty, Astrophys. J. 512, 288 (1999).
22. M. Mouchet, S. A. Ilovaisky, and C. Chevalier, Astron. Astrophys. 90, 113 (1980).

23. J. B. Hutchings, A. P. Cowley, and D. Crampton, Astrophys. J. 229, 1079 (1979).

24. C. D. T. Ash, A. P. Reynolds, P. Roche, et al., Mon. Not. R. Astron. Soc. 307, 357 (1999).

25. J. B. Hutchings, D. Crampton, and A. P. Cowley, Astrophys. J. 225, 548 (1978).

26. R. L. Kelley, J. G. Jernigan, A. Levine, et al., Astrophys. J. 264, 568 (1983).

27. P. S. Osmer and W. A. Hiltner, Astrophys. J. 217, 186 (1977).

28. J. B. Hutchings, D. Crampton, A. P. Cowley, and P. S. Osmer, Astrophys. J. 217, 186 (1977).

29. A. P. Reynolds, R. W. Hilditch, S. A. Bell, and G. Hill, Mon. Not. R. Astron. Soc. 261, 337 (1993).

30. K. Makishima, K. Koyama, S. Hayakawa, and F. Nagase, Astrophys. J. 314, 619 (1987).

31. D. Crampton, J. B. Hutchings, and A. P. Cowley, Astrophys. J. 225, L63 (1978).

32. A. P. Reynolds, S. A. Bell, and R. W. Hilditch, Mon. Not. R. Astron. Soc. 256, 631 (1992).

33. J. van Paradijs, E. J. Zuiderwijk, R. J. Takens, and G. Hammerschlag-Hensberge, Astrophys. J., Suppl. Ser. 30, 195 (1977).

34. O. Barziv, L. Kaper, M. H. van Kerkwijk, et al., Astron. Astrophys. 377, 925 (2001).

35. J. B. Hutchings, Astrophys. J. 235, 413 (1980).

36. D. Crampton, J. B. Hutchings, and A. P. Cowley, Astrophys. J. 299, 839 (1985).

37. J. B. Hutchings, Astrophys. J. 192, 685 (1974).

38. E. A. Antokhina, A. M. Cherepashchuk, and V. V. Shimanskiı̆, Izv. Ross. Akad. Nauk, Ser. Fiz. 67, $293(2003)$.

39. A. A. Rubashevskiü, Astron. Zh. 68, 799 (1991) [Sov. Astron. 35, 626 (1991)].

40. H. Quantrell, A. J. Norton, T. D. C. Ash, et al., Astron. Astrophys. (2003, in press).

41. L. Kaper, G. Hammerschlag-Hensberge, and E. J. Zuiderwijk, Astron. Astrophys. 289, 846 (1994).

42. D. J. Hudson, Statistics. Lectures on Elementary Statistics and Probability Theory (Geneva, 1964; Mir, Moscow, 1970).

43. F. Nagase, R. H. D. Corbet, C. S. R. Day, et al., Astrophys. J. 396, L147 (1992).

44. A. M. Cherepashchuk, Astron. Zh. 70, 1157 (1993) [Astron. Rep. 37, 585 (1993)].

Translated by N. Samus' 